\title{
Vitamin-anticancer drug conjugates: a new era for cancer therapy
}

\author{
Ritesh P. Bhole ${ }^{1}$ (D), Shradha Jadhav ${ }^{1}$ (D), Yogesh B. Zambare ${ }^{1}$ (D), Rupesh V. Chikhale ${ }^{2}$ (D), \\ Chandrakant G. Bonde ${ }^{3}$ (i) \\ 1Dr. D. Y. Patil Institute of Pharmaceutical Sciences and Research, Department of Pharmaceutical Chemistry \& \\ Pharmaceutical Quality Assurance, Pune, India \\ ${ }^{2}$ University of East Anglia, Faculty of Pharmacy, Norwich Research Park, United Kingdom \\ ${ }^{3}$ SPTM, School of Pharmacy, Department of Pharmaceutical Chemistry, Shirpur, India
}

ORCID IDs of the authors: R.P.B. 0000-0003-4088-7470; S.J. 0000-0002-6443-5009; Y.B.Z. 0000-0001-5115-0971; R.V.C. 0000-0001-5622-3981; C.G.B. 0000-0001-5712-1119

Cite this article as: Bhole, R. P., Jadhav, S., Zambare, Y. B., Chikhale, R. V., \& Bonde, C. G. (2020). Vitamin-anticancer drug conjugates: A new era for cancer therapy. Istanbul Journal of Pharmacy, 50 (3), 312-322.

\begin{abstract}
Background: Following cardiovascular diseases, cancer is the world's second leading cause of death. Chemotherapy is the conventional gold technique for successful treatment of cancer. There are some drawbacks associated with traditional chemotherapy, namely, low aqueous solubility, limited biological half-life, production of multidrug resistance and non-specificity (lack of targeting ability) or dose-limiting cellular toxicity. To develop a targeted drug delivery for its anticancer effect is still a challenging task.

Methods: We developed literature review methods which included inclusion and exclusion criteria for identifying potentially relevant articles, articles search strategies, abstract review protocols and a comprehensive scoring system for published studies. This study contains a detailed survey of various reported methods such as folic acid-drug conjugates, CobalaminDrug Conjugate, Vitamin B12-Conjugated and Paclitaxel-Loaded Micelles etc., all of which were studied for their methods of preparation and possible impact on biological activity.

Results: Due to its specific ability to carry anticancer drugs directly to tumours, vitamin-mediated drug targeting has recently emerged as a novel concept. Solid tumour cancer has an unquenchable appetite for various essential vitamins, resulting in over-expression of the receptors involved in cell internalization of vitamins on the surface of cancer cells. So, the vitamin drug conjugates are specifically important for carrying the anticancer drugs directly to the tumour cells. Biotin, folic acid, vitamin B12 and riboflavin, the vitamin necessary for the division of all cells, especially cancer cells, have recently been examined as targeting agents.

Conclusion: Vitamin-Drug Conjugate methods were found to be the most suitable methods amongst all the other reported methods and they can be applied for current therapy against cancer.
\end{abstract}

Keywords: Cancer, chemotherapy, anticancer drug and vitamin

\section{INTRODUCTION}

Cancer is the unchecked development of cells in the human body and these cells have the capacity to metastasize. If this spread is not managed, cancer will lead to death (Davis, 2019). It is a multicellular and multigenic disease that may occur with a complex etiology from any type of cell and organ (Baskar,
Lee, Yeo, \& Yeoh, 2012). Cancer has become the world's second-largest cause of death after cardiovascular disease (Padma, 2015).

Mainstream modalities of treatment include surgery, radiotherapy, immunotherapy, hormonal therapy, laser therapy, and stem cell therapy. Surgery and radiotherapy are the most effec- 
tive and common therapies for localized primary tumors and non-metastatic cancers. Cancer drugs (chemotherapy, biological and hormonal therapy) are used to treat metastatic cancer. Drugs spread to all tissues in the body, through the bloodstream (Pérez-Herrero, \& Fernández-Medarde, 2015). Thus, for effective cancer treatment, chemotherapy is said to be as the gold standard strategy. Chemotherapy is based on chemotherapeutic drugs (i.e. toxic compounds) that act by inhibiting the cancer cells' rapid proliferation. There are some drawbacks associated with traditional chemotherapy, i.e. chemotherapeutic drugs with cancer cell suppression, which can also prevent the rapid growth needed to maintain normal cells, such as bone marrow, gastrointestinal tract and hair follicle cells, leading to undesirable side effects in cancer treatments (Seifu \& Nath, 2019). Some other drawbacks in cancer treatment associated with free chemotherapeutic agents are low aqueous solubility, short biological half-life, production of multidrug resistance and non-specificity (lack of targeting capacity) or dose-limiting cellular toxicity.

Consequently, it is still difficult to target an anti-cancer drug selectively to cancerous cells and to achieve a breakthrough in cancer research. To achieve this goal, different approaches can be implemented. The most popular is to provide an effective carrier for a medication. Thanks to its ease of use and uncomplicated chemical modification, polymeric drug carriers are the most powerful (Pawar, Badhwar, Kharas, Khandare, \& Vavia, 2012; Tripodo, Mandracchia, Collina, Rui, \& Rossi, 2014). Such systems usually have the ability to accumulate by means of passive drug targeting in different organs as a result of their removal route or for active organotropism, and a more specific drug targeting can be accomplished by adding a carrier with specific targeting agents, such as vitamins, antibodies, peptides, magnetic particles or hormones (Cavallaro, Maniscalco, Campisi, Schillaci, \& Giammona, 2007; Bareford, Avaritt, Ghandehari, Nan, \& Swaan, 2013; Tripodo et al., 2014; Gibiansky \& Gibiansky, 2014; Qu, Zhou, Chen, Chen, \& Shen, 2015). The most commonly used method for drug targeting to specific cells is to attach a drug directly to targeting moiety to form a new chemical entity which is pharmacologically active, i.e. prodrug (Elsadek et al., 2010; Tripodo et al., 2014). In these conditions, the so-called vitamin-mediated drug targeting has recently emerged as a new concept for carrying anticancer drugs particular to tumours (Mahato, Tai, \& Cheng, 2011; Bildstein, Dubernet, \& Couvreur, 2011; Fortin \& Berube, 2013). As it is well known, all living cells require vitamins for their survival, whereas rapidly dividing cells that are found in solid tumour cancer, have an unquenchable appetite for different essential vitamins, resulting in vitamin receptors being over-expressed on the surface of cancer cells. In addition, there has been a claim that the combination of an anticancer drug with a particular vitamin contributes to the development of vitamin-drug conjugates (Figure 1). This vitamin drug conjugate would lead to the targeting of a higher amount of drugs, i.e. a high dose to the targeted cancer cells. Biotin, folic acid, vitamin B12 and riboflavin, which are necessary for the division of all cells, in particular cancer cells, have recently been investigated as targeting agents (Chen et al., 2010; Russell, McTavish, \& McEvan, 2011).

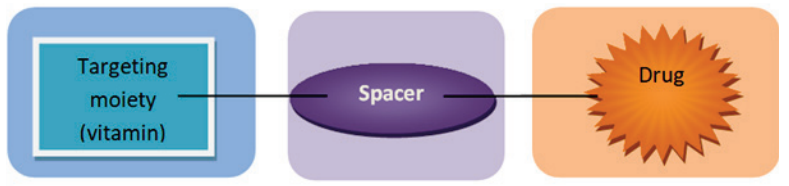

Figure 1. Vitamin drug conjugate.

\section{Vitamin-Drug Conjugates}

A drug is linked directly or through a spacer with a targeting moiety to form a pharmacologically active new chemical entity to produce vitamin-drug conjugate.

Advantages: Vitamin drug conjugates will be nontoxic, and they will be specifically internalized into cancerous cells and release anticancer drugs without loss of potency. In addition, they will minimize the systemic toxicity by being stable in blood circulation, and provide a target specific activity by sparing the normal cell that will minimize any side effects (Ojima, Zuniga, Berger, \& Seitz, 2012).

\section{Vitamin-9 (Folic Acid)-Drug Conjugates}

Folic acid (FA) is more closely related to folate receptors (FR). Glycosylphosphatidylinositol (GPI)-linked membrane folate receptor was involved in folate-based chemotherapeutic drug receptor-mediated absorption (Rijnboutt, Jansen, Posthuma, Hynes, \& Schornagel, 1996). This GPI-connected membrane protein collects the ligands from the extracellular matrix and transports them through the recycled endosomal pathway to the intracellular space (Figure 2). The folate receptors are also recognized as tumour antigens/biomarkers due to the fact that the folate receptors are commonly absent in normal tissues. This has led to the development of therapeutics and diagnosis approaches for the treatment of cancer (Leamon et al., 2007). Hence molecular payloads with a size range from radionuclides to large constructs of DNA and liposome have been

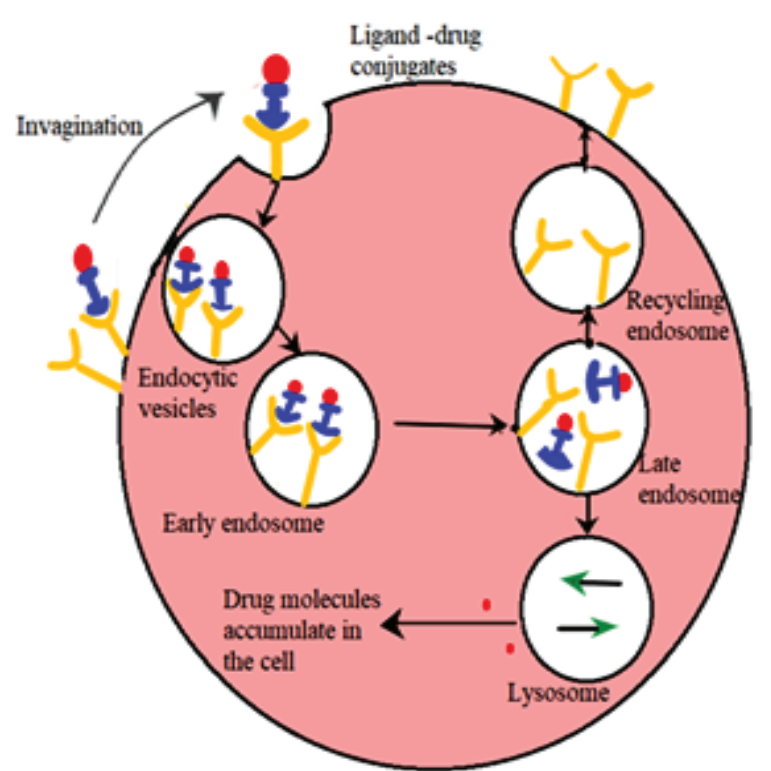

Figure 2. Receptor mediated endocytosis of folate-drug conjugate. 
successfully delivered through the FR pathway within cancer cells. One particularly related approach is to attach powerful chemotherapy drugs to FA to form a small molecule drug conjugate or SMDCs. (Vlahov \& Leamon, 2012).

\section{Folic acid-paclitaxel conjugates}

Yuxuan et al., 2019, engineered the conjugates of folic acidpeptide-paclitaxal (FA-P3/P7-PTX), to resolve drug resistance, achieve targeted delivery to tumours, increase cellular absorption, and allow water-soluble conjugates. The synthesized (FAP3/P7-PTX) conjugate was a folic acid-lytic peptide conjugate, in which lytic peptides I-3 and I-7 were used as molecular carriers and cell-disrupting peptides. 16-site cysteine-substituted I-3 and I-7 were named as P3 and P7, respectively, and served as a peptide backbone. The conjugates were tested for their efficacy and also showed higher anti-proliferative activity than free paclitaxel in MCF-7/PTX cells. Higher cellular uptake in MCF-7/PTX cells was shown by, FA-P3-PTX than P3-PTX, which was based on folate receptors present. FA-P7-PTX had a much stronger effect on cell toxicity, apoptosis and membrane disturbance behavior in MCC. Similar to FA-P3-PTX, FA-P7-PTX had more ability to suppress tumor growth than PTX. (Wang et al., 2011; Gaspar, Costa, Qeiroz, Pichon, \&Souse, 2015; Dai et al., 2019).

\section{Folic acid-DAVLBH conjugates (Vintafolide)}

Vintafolide (formerly EC145), the most active FA-SMDC, developed by Leamon et al., 2014, was a water-soluble conjugate that selectively delivers the medication desacetyl vinblastine monohydrazine (DAVLBH) to tumours over-expressed to FRalpha (Leamon et al., 2014). Preclinical studies have shown a higher affinity of vintafolide to bind to FRa, and therefore have a very strong and selective action against FRa-positive xenografts as compared to untargeted DAVLBH (Vlahovet al., 2009).

Vintafolide consists of 4 part modules: 1) a folic acid moiety targeting FRa, 2) a hydrophilic peptide spacer, 3) an autoimmolative disulfide linker, and 4) a DAVLBH microtubule-stabilizing compound (Figure 3).

Leamon et al., 2014, conducted a study to assess the impact of changing three of the vintafolide's constituent elements out of the four. It was noted that by changing the spacer composition, given the spacer remained hydrophilic, the vintafolide's potency was minimally affected. By comparison, the bioreleasable linker such as glutathione (GluSH) in the endosomal setting is cleaved by the intracellular thiols which is of critical importance for the conjugate action and has been the most successful approach to activated drug release within the cell (Figure 4). As an example, self-immolative disulfide and acyl hydrazone were exercised both in vitro and in vivo activity, while vintafolide analogs showed more stable amide linkers and thioether linkers did not show (Leamon et al., 2014). In addition, it was shown that by replacing DAVLBH with other clinically approved vinca alkaloid drugs (such as vincristine, vinflunine, vinorelbine and vindesine) while retaining the cleavable disulfide linker, vintafolide was the only variant showing biological activity in vitro and in vivo. It was later hypothesized that other absence of activity in other vinca alkaloid was found due to alteration in the chemical structures, following disulfide reduction and linker release.

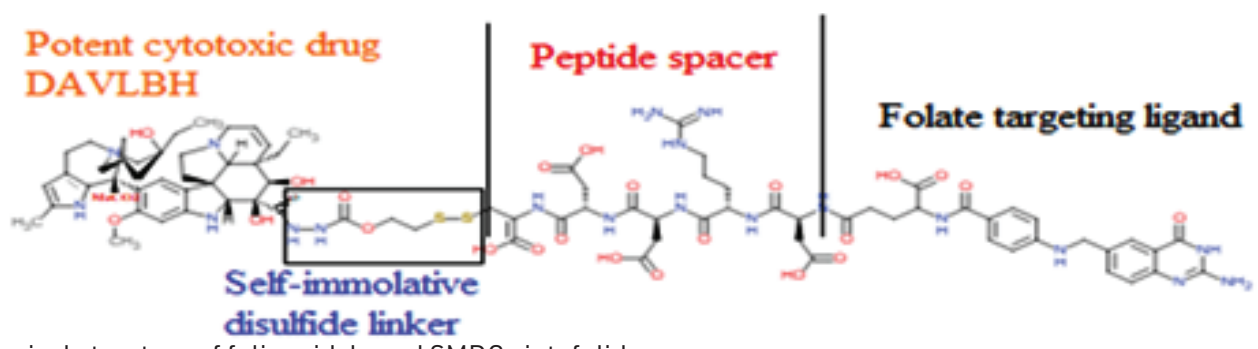

Figure 3. Chemical structure of folic acid-based SMDC vintafolide.
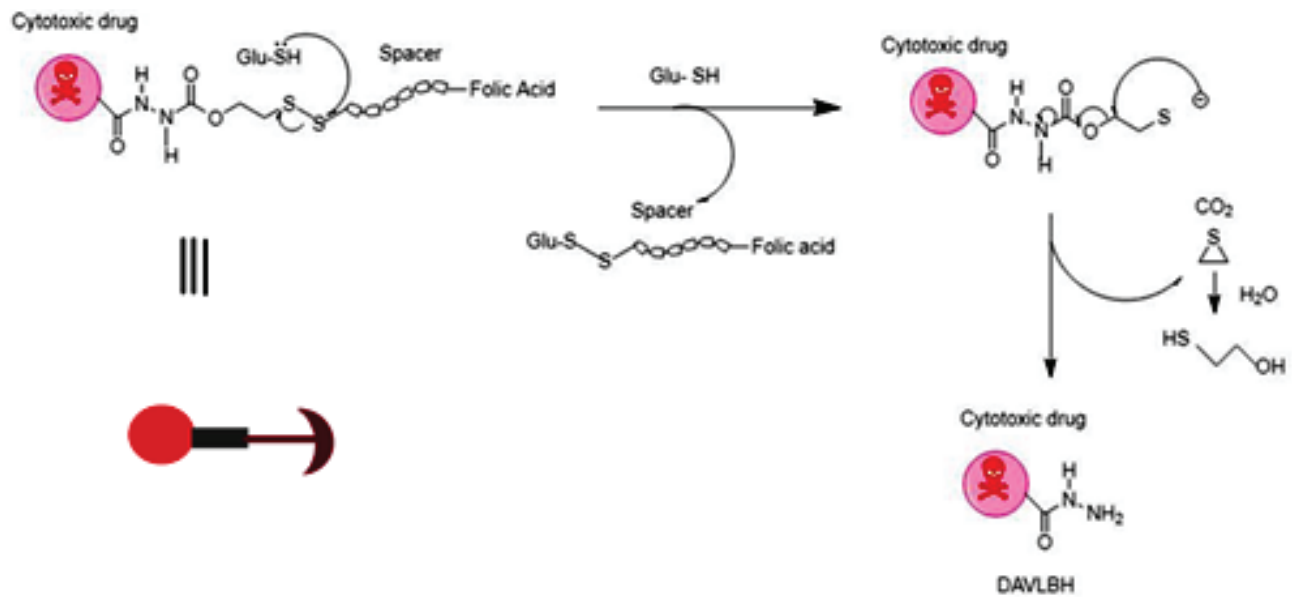

Figure 4. Drug release mechanism of vintafolide. 
EC145 was the first clinically tested FA-SMDC. Vintafolide showed reliability, both as a single agent and in conjunction with doxorubicin, in two phase II tests (ovarian and non-small cell lung cancers) and in a controlled open-label Phase II test (platinum-resistant ovarian cancer). It entered the clinical trials phase-III for advanced platinum-resistant ovarian cancer in 2010 (Leamon et al., 2014). This FA-SMDC failed to meet the previously stated progression-free survival requirements and the trial was prematurely terminated due to this outcome. Despite expectations of doing otherwise, the Phase III trials were unable to demonstrate the dominance of the targeted FR therapy over conventional chemotherapy. Future studies and experiments therefore need to be performed for a good range of qualifying patients who may adequately benefit from antiFR therapy. (Leamon et al., 2014 Sausville et al., 2007; Reddy et al., 2007; Naumann et al., 2013; Naumann \& Coleman, 2011; Vlahov et al., 2009; Fernandez, Javaid, \&Chudasama, 2018).

\section{Folate-taxoid conjugates}

Seitz, 2015, developed a folate-taxoid conjugate of the next decade that is highly potent for use against drug-sensitive and drug-resistant cell lines of cancer. This folate-taxoid conjugate (Figure 5) contains both a highly potent taxoid, i.e an analog of the chemotherapeutic drug Taxol, and also folic acid moiety. This SMDC has a hydrophilic PEGylated dipeptide spacer and an autoimmolative disulfide linker, similar to vintafolide (Seitz, Vineberg, Herlihy, Park, \& Melief, 2015).

In vitro research was conducted to compare the behavior of the free taxoid and taxoid conjugate in FRa-positive and FRanegative cells. For both cell lines, i.e., free (taxoid) SB-T-1214 was highly potent as expected. Moreover, taxoid conjugate demonstrated significant cytotoxicity to the FRa-positive cell lines. In addition, taxoid conjugate also showed more than a 1000-fold decline in toxicity to normal cells compared to the free drug (Fernandez et al., 2018).

Such FA-SMDCs are from a vast field of conjugates using a disulfide linker for cytotoxic drug release. It is particularly relevant to note that folate is conjugated to many other medications, such as camptothecins (Henne, Kularatne, Hakenjos, Carron, \& Henne, 2013), tubulysins (Reddy et al., 2009; Bartouskova, Melichar, \& Mohelnikova, 2015), mitomycins (Leamon et al., 2007), and maytansinoids, all of which were prepared and evaluated by disulfide linker.

\section{5-Fluorouracil loaded PLGA-1, 3-diaminopropane-folic acid nanoparticles}

Because of a low FA conjugation ratio, (Wang et al., 2015), developed a PLGA [poly (lactic co-glycolic acid)]-based drug delivery carrier with a targeting mood such as folic acid (FA) that has poor targeting performance. A crosslinker 1, 3-diaminopropane was used in this work to produce a FA-conjugated PLGA device and achieved a high 46.7\% (mol / mol) conjugation level. The prepared biomaterial based on PLGA was then used for encapsulation into nanoparticles of 5-fluorouracil (5-FU). The IC50 of 5-FU loaded PLGA-1, 3-diaminopropanefolic acid nanoparticles on HT-29 cancer cells was observed to be $5.69 \mathrm{mg} / \mathrm{mL}$ in vitro experiments and is smaller than that of 5-FU loaded PLGA nanoparticles and 5-FU with only 14.17 and $22.9 \mathrm{mg} / \mathrm{mL}, I C_{50}$ respectively. The images of fluorescent microscopy showed that targeting nanoparticles have more affinity with cancer cells, and nanoparticles with FA are taken up in greater amount than pure drugs and untreated nanoparticles, by the cancer cells with HT-29. The 5-FU primed PLGA-1, 3-diaminopropane-folic acid nanoparticles are therefore one of the highly efficient methods for the targeted delivery of the drug to the cancer cells (Wang, Li, Chen, Gao, Zeng, \& Kong, 2015).

\section{Folic acid- trimethyl chitosan- paclitaxel conjugates}

He \& Yin, 2017, developed a trimethyl chitosan (TMC-PTX) conjugated paclitaxel (PTX) modified with folic acid (FA), (FA-TMCPTX) for oral and intravenous delivery of PTX. Modification of FA has been carried out to alleviate conjugate protein adsorption (Pawar et al., 2012; Henne et al., 2013, He \& Yin, 2017).

\section{Folic acid coupled to the pegylated-liposomes of 5-fluorouracil}

Pegylated-liposomes coupled with folic acid were produced by Gupta et al., 2007, and they increased the in vitro absorption by up to 11 times compared to non-coupled pegylated liposomes. The tumor-inhibitory effect of FA-SL (stabilized liposome) was significantly greater than that of free 5-FU and SL. Therefore, this device can be successfully used to target 5-FU to the tumour cells. (Gupta, Jain, Jain, \& Jain, 2007).

\section{Abrabinogalactan-folic acid- drug conjugate}

The FA-AG-GFLG-MTX conjugate, illustrated by Pinhasi et al., 2009, shows the distribution of a cytotoxic cargo to FR-overexpressing cells. They also showed, a target-activated release

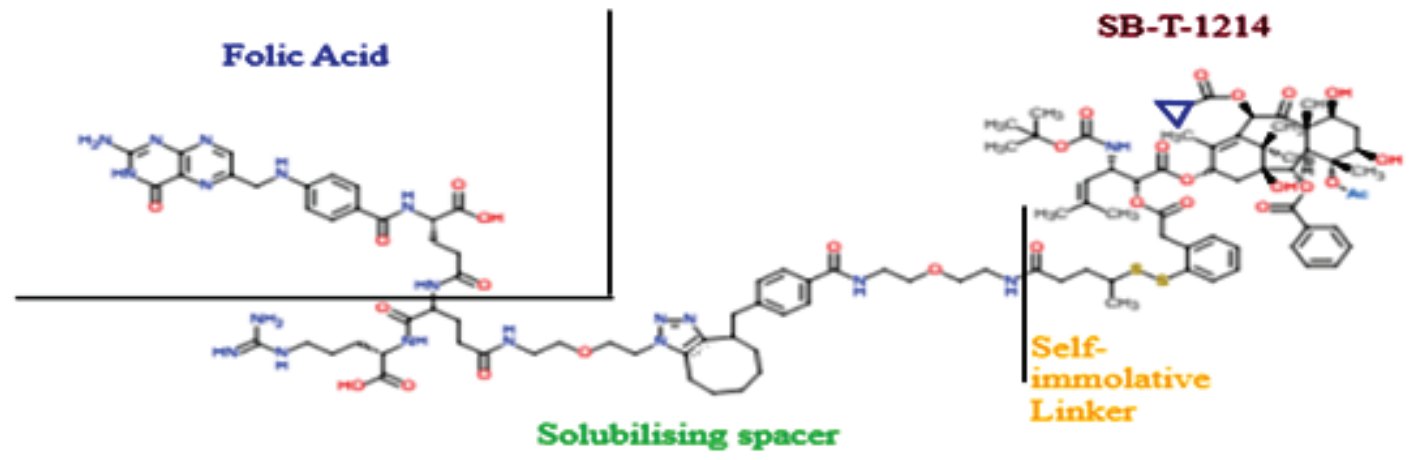

Figure 5. Structure of folate- taxoid conjugate. 
mechanism by adding an endosomally cleavable peptide (GFLG) into the conjugate. Compared to normal cells, this conjugate showed a 6.3-fold increase in cytotoxic activity of cancer cells. This result creates a novel FA bound polymer nanoconjugate to transmit methotrexate to over-expressed cancer cells in FR. (Pinhassi et al., 2009).

\section{Cobalamin-Drug-Conjugate}

The invention provides a cobalamin-drug conjugate in which cobalamin is indirectly covalently bound to an anti-tumour drug through a cleavable linker and one or more optional spacers, and is suitable for the treatment of tumour-related diseases. Cobalamine is covalently bound by the $5^{\prime}-\mathrm{OH}$ of the cobalamin ribose ring to a cleavable linker or the first spacer. The drug is connected to the cleavable linker's Second Spacer through an additional or current functional group on the surface. This conjugate then forms a transcobalamin complex (any of its isoforms). This complex binds to the receptor present on the cell membrane and is the result of cellular internalization, and the intracellular enzymes then separate the conjugates and activate the drug. Based on the conjugate form, the specific class or type of intracellular enzyme influences the cleavage. As there is a high demand of rising cells for cobalamine, tumour cells have more appetite than the normal cells. Compared to a free drug, the technology conjugate has an increased potency and reduced systemic toxicity. (Jung \& Keller, 2012).

\section{VB12-Conjugated and PTX-Loaded Micelles (VB12-Seri- cin-PBLG (Poly (Gamma-Benzyl-L-Glutamate))-PTX)}

Guo et al., 2018, introduced sericin micelles to reverse drug resistance in their previous research, but these sericin micelles could not selectively bind to the cells of gastric cancer (GC). They developed sericin micelles conjugated with vitamin B12 (VB12) for the targeted treatment of gastric cancer. Researchers studied their physicochemical properties, the function of antitumours, and the cellular uptake. It was shown that VB12sericin-PBLG-PTX micelles have an acceptable particle size, have strong dispersion, and are bio-safe. Through following transcobalamin-II (CD320)-receptor-mediated endocytosis, the micelles were internalized cellularly with gastric targeting and improved cellular uptake capacities, they reversed drug resistance, and modified the mitochondrial transmembrane / apoptosis pathway (Guo et al., 2018).

\section{Vitamin B12-Metal Conjugates for the Therapeutic and Diagnostic Application}

Vitamin B12 (cyanocobalamin) is an essential nutrient characterized by very poor bioavailability. Due to the rapid rate of proliferation of cancerous cells, they have a greater demand of nutrients including a greater vitamin B12 uptake compared to normal cells. Such a cyanocobalamin appetite can be used for site-specific delivery of the medication to tumour cells by conjugating the vitamin B12 (carrier) anticancer drug for therapeutic use or by conjugating a fluorophore or radionuclide diagnostic agent with vitamin B12 for diagnostic use. Vitamins such as B12 are transformed through biologically active cofactors, i.e. methylcobalamin (which is used to manufacture methionine) and adenosylcobalamin (which is used as a coenzyme to generate energy through the synthesis of carboxylic acids), and it is noteworthy that the quickly dividing tumour cell needs higher amounts of methionine and energy for replication, allowing vitamin B12 to be more aimed at cancer.

\section{Therapeutic vitamin B12- metal conjugates}

An impressive number of studies on platinum-based and other metal-based anticancer drugs were generated from the marketing and clinical establishment of anticancer drug cisplastin, but the main drawback associated with such treatment is low water solubility, low bioavailability, non-specific tumour cell binding, and higher toxicity. There has lately been a greater interest in the development of vitamin-metallodrug conjugates, but none of them have yet reached the clinical trial stage and have thus far only explored their therapeutic efficacy.

Platinum: Florea \& Büsselberg, 2011, developed a strategy to address the issue of non-specific anticancer medication binding, such as cisplastin, and its severe side effects. Conjugating cisplastin to cyanocobalamin as an anticancer medication has been speculated as a possible way of improving tumourspecific attachment with a better clinical output. The metal containing scaffolds are directly linked by the nitrogen atom to the cyanogroup present on vitamin B12, producing a heterodinuclear derivative (B12-Coll-CN-M), in which the present vitamin serves as a ligand. For vitamin B12 to be transformed into its cofactor (methylcobalamin or adenosylcobalamin) requires first of all the reduction of Co (III) to Co (II) by removing the main cyanogroup. Thus, when mixing a metal product with the moiety B12-Colll-CN, the release of the cyanometal species (CN-M) will occur directly within the cells, so this vitamin B12-metal conjugate would be considered a prodrug.

In vitro cytotoxicity experiments on human ovarian adenocarcinoma A2780 cells and human breast adenocarcinoma MCF7 cells have shown that the isolated Pt (II)-cyanoderivative has a comparable anti-cancer action to cisplastin, thereby indicating that conjugate (B12-Coll-CN-PtII) can be identified as a prodrug because it has the ability to release Pt II, a cytotoxic agent, directly into the body. Unfortunately, this hypothesis was not consistent with evidence that the initial conjugate was less cytotoxic than free cisplastin. (Ruiz-Sanchez, Konig, Ferrari, \& Alberto, 2011; Tran, Sturup, Lambert, Gammelgaard, \& Furger, 2016; Pettenuzzo, Pigot, \& Ronconi, 2017).

\section{Diagnostic vitamin B12-metal conjugates}

Recent developments for diagnostic applications of vitamin B12-metal conjugates using radiodiagnostic or fluorescent samples are discussed below. A variety of biomolecules are used as a carrier for radionuclides, some of which have been approved by Food and Drug Administration, such as peptides and monoclonal antibodies. Among all of these, vitamins were researched as a target-specific delivery carrier in which vitamin B12 is the least studied.

Gadolinium: -Gadolinium (III) is a paramagnetic material that is used for magnetic resonance imaging (MRI) and may also be cytotoxic. Siega et al., 2009, formed two metal chelating ligands of vitamin B12, either diethylenetriamine-N, N, 
N',N",N",N"-pentaacetic acid (DTPA) or triethylenetetramine-N, N",N",N"N",N",N"',N"'-hexaacetic acid (TTHA), and the corresponding gadolinium(III) combines Gd-1 and Gd-2 (Figure 6).

On human immortalized myelogenous leukemia K562 cells, in vitro cytotoxicity was performed; in which it was found that the Gd-1 conjugate emitted the $\mathrm{Gd}^{3+}$ molecule, resulting in reduced cell viability. On the other hand, when the same test was performed of more stable Gd-2 ion, it did not show $\mathrm{Gd}^{3+}$ cell internalization, and also no significant influence was observed on cell viability (Siega et al., 2009; Pettenuzzo et al., 2017).

\section{Vitamin D3-Drug Conjugates}

Patil, Gawali, Patil, \& Basu, (2014), developed nanoparticles of vitamin D3 to deliver some of the commonly used and clinically licensed anticancer drugs such as doxorubicin (a DNA-damaging agent), paclitaxel (a microtubule-stabilizing agent) and PI103 (a phosphatidylinositol-3-kinase inhibitor). Next, they developed vitamin D3-drug conjugates (Figure 7-compound no 3, 4 and 5), then the biocompatible vitamin D3 nanoparticles were synthesized from these conjugates using a process of hydration-extrusion solvent evaporation-lipid film (Figure 8).

Such vitamin D3-nanoparticles were examined in the drug release model. A gradual and continuous release of medications over a period of time and an accelerated release was found at $\mathrm{pH} 5.5$ relative to $\mathrm{pH}$ 7.4. Connections in vitamindrug conjugates, i.e. vitamin-D3-PI103 conjugates, contain phenolic-ester connections, vitamin D3-paclitaxel conjugates contain ester connections, and amide-connected vitamin-D3-doxorubicin conjugates were more labile at $\mathrm{pH}$ 5.5 relative to $\mathrm{pH} 7.4$, resulting in increased drug release at acid $\mathrm{pH}$. In addition, the cellular absorption process of vitamin D3-NPs was studied in HeLa cells by treating them with vitD3-Dox-NPs. It was observed that internalization in HeLa cells was through a low pH lysosomal compartment, whereas free drug doxorubicin was internalized through the diffusion path. A similar endocytosis was expected for VitD3-paclitaxelNPs and vitD3-PI103-NPs (Patil et al., 2014; Petros \& DeSimone, 2010; Rajendran, Knolker \& Simons, 2010; Iversen, Skotland, \& Sandvig, 2011).

\section{Dual drug loaded vitamin D3 nanoparticles}

One of cancer chemotherapy's most challenging problems is combating drug resistance. Drug cocktails can overcome drug resistance. Double vitamin D3 nanoparticles (i.e. a fair mixture of PI103 and doxorubicin or paclitaxel or cisplatin) were developed by Palvia et al. 2014, (Table 1). Nanoparticles dual drug release was observed to be higher at $\mathrm{pH} 5.5$ relative to $\mathrm{pH} 7.4$ at a slow rate over 72 hours in a prolonged fashion, and at $37^{\circ} \mathrm{C}$ and $40^{\circ} \mathrm{C}$ for more than 15 days. Cell death by dual drug loaded nanoparticles in human hepatocellular<smiles>CC1(C#N)CCCC1COC(=O)CN(CCN(CCN(CC(=O)O)CC(=O)O)CCN(CC(=O)O)CC(=O)O)CC(=O)O</smiles>

(1)<smiles>COC1(C#N)CCCC1OC(=O)CN(CCN(CCN(CCN(CC(=O)O)CC(=O)O)CC(=O)O)CC(=O)O)CC(=O)O</smiles>

(2)

Figure 6. Vitamin B12-DTPA and vitamin B12-TTHA ligand.

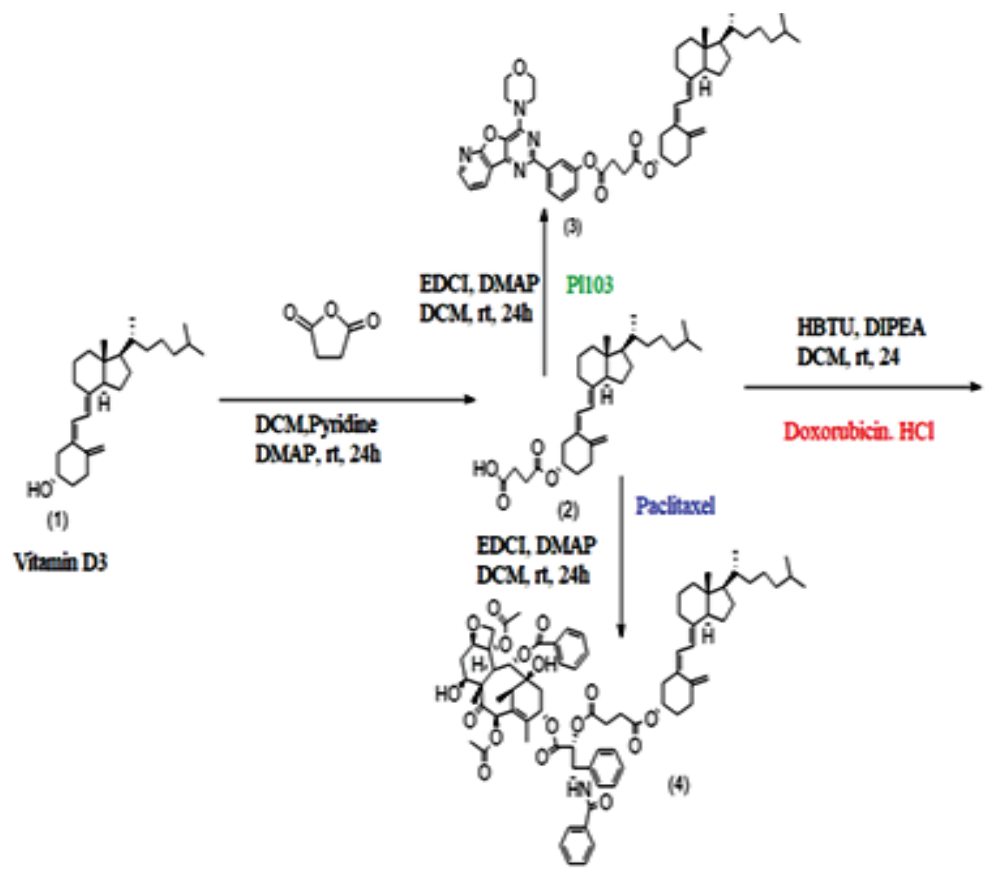

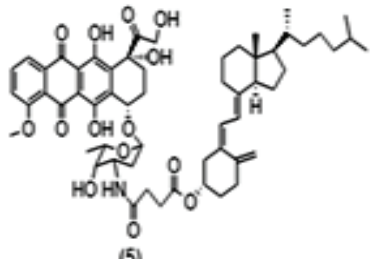

(5)

Figure 7. Synthetic scheme for vitamin D3- drug conjugate. 

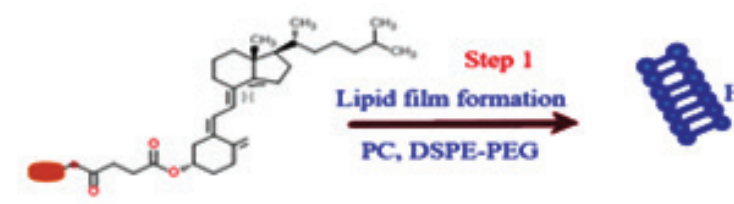

Step 2

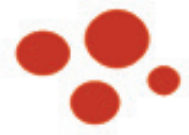

Vitamin D3 drug conjugates

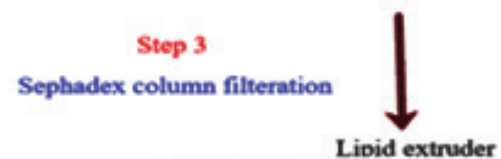

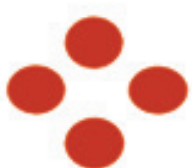

Drug loaded vitamin D3-nanoparticle

Figure 8. Synthetic scheme for vitamin D3 nanoparticles.

\section{Table 1. Dual drug loaded vitamin-D3 nanoparticles and its application.}

\begin{tabular}{|ll|}
\hline NANOPARTICLES & APPLICATION \\
\hline $\begin{array}{l}\text { VitD3-PI103-Dox-NP and } \\
\text { VitD3-PI103-CDDP-NP. }\end{array}$ & $\begin{array}{l}\text { Showed toxicity by inducing } \\
\text { apoptosis, through DNA } \\
\text { damaging. }\end{array}$ \\
VitD3-PI103-CDDP-NP. & $\begin{array}{l}\text { Showed improved efficacy } \\
\text { in 5-fluorouracil resistant } \\
\text { Hep3B-5FU-R cells }\end{array}$ \\
VitD3-PI103-Profalvin-NP. & $\begin{array}{l}\text { Internalized cisplatin } \\
\text { resistant Hep3B-R cells } \\
\text { faster (3 mins) as compared } \\
\text { to Hep3B cells. }\end{array}$ \\
\hline (Palvai, Nagraj, Mapara, Chowdhury, \& Basu, 2014).
\end{tabular}

carcinoma (Hep3B cell) was found to be increased at 24 hours compared to monotherapy (Persidis, 1999; Garraway \& Janne, 2012; Parhi, Mohanty, \& Sahoo, 2012; Palvai, Nagraj, Mapara, Chowdhury, \& Basu, 2014).

\section{Vitamin-E-Drug-Conjugates}

\section{Vitamin-E analogue-neomycin conjugate for RNA-I drug}

Iwata et al., 2015, synthesized a vitamin E-conjugated neomycin derivative to administer RNAi (RNA interference) medication to the cell of liver cancer and tested it for its biological and physicochemical properties. SiRNA delivery studies were not entirely successful, but some of the neomycin derivatives showed significant RNAi activity in liver cancer cells. (Iwata et al., 2015).

\section{Biotin-Drug-Conjugates}

\section{Biotin-taxoid conjugates}

Chen et al., 2010, developed a biotin (vitamin or vitamin B7)taxoid conjugate for a tumour-targeted drug delivery system.These conjugates included biotin as a mood-targeted tumour, a mechanism-based self-immolative linker, and a cytotoxic agent (SB-T-1214) (Figure 9). They developed a tumour-specific mechanism for the delivery of drugs based on endocytosis mediated by vitamin receptors. Reports of L1210FR leukemia cells that over-express biotin receptors were performed. The result was an excellent site-specific delivery of the conjugates, reducing unwanted toxicity to healthy cells (Chen et al.,2010; Tripodo, Mandracchia, Collina, Rui, \& Rossi, 2014)

\section{Biotin-doxorubicin conjugates}

Ibsen et al., 2010, developed and synthesized the biotindoxorubicin conjugate in which the amine component of doxorubicin was derivatized with a photocell biotinylated spacer (Figure 10) (Tripodo et al., 2014).

Ibsen et al., 2010, demonstrated that the doxorubicin (i.e. active drug) is only released after the conjugates are internalized into the cancerous cells and then further activated via exposure to UV at $350 \mathrm{~nm}$ of the photocleavable group in the conjugate, leading to a decrease in the cytotoxic effects on normal cells.

The cell proliferation assay results clearly evidenced a lower cytotoxicity of conjugate than that of free doxorubicin (Sutherland \& Griffin, 1981; Ibsen et al., 2010).

The brief summary of all the reported methods and their advantages over conventional chemotherapy is shown in Table 2.

Figure 9. Chemical structure of biotin-SBT-1214-fluorescein conjugate. 


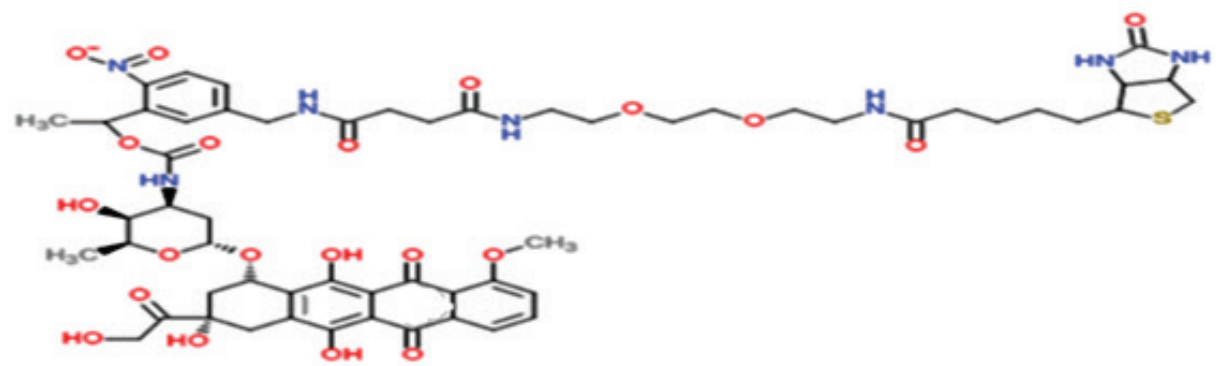

Figure 10. Chemical structure of biotin-doxorubicin conjugate.

Table 2. Vitamin-drug conjugates and their advantages over chemotherapeutic drugs.

Sr. No. Method Advantages over chemotherapeutic drugs

1. Folic acid-Paclitaxel Conjugates.

Conjugate showed higher anti-proliferative activity, Higher cellular uptake than free paclitaxel in MCF-7/PTX cells. FA-P7-PTX had a much stronger effect on cell toxicity, apoptosis and membrane disturbance behavior in MCC. FA-P3-PTX, FA-P7-PTX conjugates had more ability to suppress tumour growth than PTX. (Dai et al., 2019).

2. Folic acid-DAVLBH (desacetyl vinblastine Vintafolide showed higher affinity to bind FRa (folate receptormonohydrazine) conjugate / Vintafolide. alpha), and therefore has a very strong and selective action against FRa-positive xenografts as compared to untargeted DAVLBH. (Vlahov et al., 2009).

3. Folate-Taxoid Conjugates.

Taxoid conjugate demonstrated significant cytotoxicity to the FRapositive cell lines. In addition, taxoid conjugate also showed more than 1000-fold decline in toxicity to normal cells compared to the free drug (Fernandez et al., 2018).

4. 5-Fluorouracil Loaded PLGA1, 3-Diaminopropane-Folic Acid Targeting nanoparticles have more affinity with cancer cells and nanoparticles with FA are taken up in greater amount than pure Nanoparticles drugs and untreated nanoparticles, by the cancer cells with HT-29. (Wang et al., 2015).

5. Folic Acid- Trimethyl Chitosan- Paclitaxel Conjugates

Folic acid modification in TMC-PTX alleviated the protein adsorption of conjugate. FA-TMC-PTX conjugate showed enhanced antitumor activity. (He \& Yin, 2017; Pawar et al., 2012; Henne et al., 2013).

6. Folic Acid Coupled to the PegylatedLiposomes of 5-Fluorouracil

Pegylated-liposomes coupled with folic acid showed increased in-vitro absorption by up to 11 times compared to non-coupled pegylated liposomes. The tumour-inhibitory effect of FA-SL (stabilized liposome) was significantly greater than that of free 5-FU and SL. (Gupta et al., 2007).

7. Abrabinogalactan-Folic Acid- Drug Conjugate.

This conjugate showed a 6.3-fold increase in cytotoxic activity of cancer cells compared to the normal cells (Pinhassi et al., 2009).

8. Cobalamin-Drug Conjugate.

As there is a high demand of rising cells for cobalamine, tumor cells have more appetite than the normal cells. Compared to a free drug, the technology conjugate has an increased potency and reduced systemic toxicity. (Jung \& Keller, 2012).

9. VB12-Conjugated And PTX-Loaded Micelles (VB12-Sericin-PBLG (Poly (Gamma-Benzyl-L-Glutamate))-PTX)

Sericin micelles could not selectively bind to the cells of gastric cancer, the vitamin B12conjugated micelles were internalized cellularly with gastric targeting and improved cellular uptake capacities, reversed drug resistance, and modifed the mitochondrial transmembrane / apoptosis pathway. (Guo et al., 2018).

Table 2. Continued. 


\section{Vitamin B12 - Metal Conjugates for the Therapeutic and Diagnostic Application}

12. Dual Drug Loaded Vitamin D3 Nanoparticles.

11. Vitamin D3- Drug Conjugates.
Therapeutic Vitamin B12- Metal Conjugates:-Platinum: -

A2780 cells and MCF7 cells have shown that the isolated Pt (II)cyanoderivative has a comparable anti-cancer action to cisplastin, thereby indicating that conjugate (B12-Colll-CN-PtII) can be identified as a prodrug because it has the ability to release Pt II (i.e. a cytotoxic agent) directly into the body. Unfortunately, this hypothesis was not consistent with evidence that the initial conjugate was less cytotoxic than free cisplastin (Ruiz-Sanchez et al., 2011).

Diagnostic Vitamin B12-Metal Conjugates:-

Gadolinium: -K562 cells, in vitro cytotoxicity was performed; in which it was found that the Gd-1 conjugate emitted the $\mathrm{Gd}^{3+}$ molecule, resulting in reduced cell viability. On the other hand, when the same test was performed of more stable $\mathrm{Gd}-2$ ion, it did not show $\mathrm{Gd}^{3+}$ cell internalization, and also no significant influence was observed on cell viability . (Siega et al., 2009 \& Pettenuzzo et al., 2017).

Vitamin D3- Drug Conjugates showed increased drug release in acidic $\mathrm{pH}$ (5.5) as compared to $\mathrm{pH}$ 7.4. Conjugates showed internalization in HeLa cells through a low pH lysosomal compartment, whereas free drug doxorubicin was internalized through the diffusion path. (Patil et al., 2014).

Nanoparticles dual drug release was observed to be higher at $\mathrm{pH}$ 5.5 relative to $\mathrm{pH} 7.4$ at a slow rate over 72 hours in a prolonged fashion, and at $37^{\circ} \mathrm{C}$ and $40^{\circ} \mathrm{C}$ for more than 15 days. Cell death by dual drug loaded nanoparticles in human hepatocellular carcinoma (Hep3B cell) was found to be increased at 24 hours compared to monotherapy (Palvai et al., 2014).

13. Vitamin-E Analogue-Neomycin Conjugate for RNA-I Drug.

SiRNA delivery studies were not entirely successful, but some of the neomycin derivatives showed significant RNAi activity in liver cancer cells. (Iwata et al., 2015).

14. Biotin-Taxoid Conjugates.

L1210FR leukaemia cells that over-express biotin receptors were performed. The result was an excellent site-specific delivery of the conjugates, reducing unwanted toxicity to healthy cells. (Chen et al., 2010).

15. Biotin-Doxorubicin Conjugates.

A lower cytotoxicity of conjugate then that of the free doxorubicin was observed. (Ibsen, Zahavy, Wrasdilo, Berns, \& Chan, 2010).

\section{CONCLUSION}

Such vitamin-drug conjugates identify and manipulate the inherent morphological and physiological distinctions between normal cells/tissues and cancerous ones. For example, cancer cells that are rapidly growing overexpress cancerspecific receptors to increase nutrient and vitamin intakes. These receptors can be used as targets for the targeted delivery of cytotoxic drugs to cancer cells via receptor-mediated endocytosis (RME). Our review focuses on the conventional treatmentof cancer and its advantages and disadvantages. It was noted that there are many novel approaches involved in overcomingthese disadvantages. Out of several formulation therapies involved,methods such as conjugation with metal ions or conjugation with several vitamins etc. The Vitamin Drug conjugates were found to be a most effective therapy. Progress in Vitamin-drug conjugates, including some of the recent vitamin-drug conjugates for target specific delivery via vitamin-receptor mediated endocytosis mechanism, reduces the undesirable toxicity to healthy cells. Some of the vitaminmetallodrug conjugates are also given, which are used for therapeutic and diagnostic applications in cancer treatment. Out of all the methods reviewed, the vitamin B12 conjugate method was found to be more specific, and also had a more positive impact on biological activity.

From the literature survey, the conjugation of vitamins with an anticancer agent is of great interest and is becoming a highly innovative approach to improving efficacy and reducing toxicity to healthy cells.

Although very few vitamin-drug conjugates have thus far been developed, this field is in constant growth, as there is a wider need for a targeted drug delivery for cancer therapy.

Peer-review: Externally peer-reviewed.

Author Contributions: Conception/Design of Study- R.P.B, C.G.B.; Data Acquisition- R.P.B., Y.B.Z.; Data Analysis/Interpretation- S.J., R.V.C., R.P.B.; Drafting Manuscript- R.P.B., S.J., C.G.B.; Critical Revision of Manuscript- R.V.C., R.P.B., Y.B.Z.; Final Approval and Accountability- R.P.B., S.J., Y.B.Z., R.V.C., C.G.B.; Technical or Material Support- R.P.B, C.G.B.; Supervision- R.P.B., S.J., Y.B.Z., R.V.C., C.G.B

Conflict of Interest: The authors have no conflict of interest to declare.

Financial Disclosure: Authors declared no financial support. 


\section{REFERENCES}

- $\quad$ Bareford, L. M., Avaritt, B. R., Ghandehari, H., Nan, A., \& Swaan, P.W. (2013). Riboflavin-targeted polymer conjugates for breast tumor delivery. Pharmaceutical Research, 30(7), 1799-812. http://dx.doi. org/10.1007/s11095-013-1024.

- Bartouskova, M., Melichar, B., \& Mohelnikova-Duchonova, B. (2015). Folate receptor: A potential target in ovarian cancer. Pteridines, 26(1), 1-2. https://doi.org/10.1515/pterid-2014-0013.

- Baskar, R., Lee, K. A., Yeo, R., \& Yeoh, K. W. (2012). Cancer and radiation therapy: Current advances and future directions. International Journal of Medical Sciences, 9(3), 193. http://dx.doi.org/10.7150/ ijms.3635.

- Bildstein, L., Dubernet, C., \& Couvreur, P. (2011). Prodrug-based intracellular delivery of anticancer agents. Advanced Drug Delivery Reviews, 63(1-2), 3-23.http://dx.doi.org/10.1016/j. addr.2010.12.005.

- Cavallaro, G., Maniscalco, L., Campisi, M., Schillaci, D., \& Giammona, G. (2007). Synthesis, characterization and in vitro cytotoxicity studies of a macromolecular conjugate of paclitaxel bearing oxytocin as targeting moiety. European Journal of Pharmaceutics and Biopharmaceutics, 66(2), 182-192. http://dx.doi.org/10.1016/j. ejpb.2006.10.013.

- $\quad$ Chen, S., Zhao, X., Chen, J., Chen, J., Kuznetsova, L., Wong, S. S., \& Ojima, I. (2010). Mechanism-based tumor-targeting drug delivery system. Validation of efficient vitamin receptor-mediated endocytosis and drug release. Bioconjugate Chemistry, 21(5), 979-987. http://dx.doi.org/10.1021/bc9005656.

- Dai, Y., Cai, X., Bi, X., Liu, C., Yue, N., Zhu, Y. ... Qian, H. (2019). Synthesis and anti-cancer evaluation of folic acid-peptide-paclitaxel conjugates for addressing drug resistance. European Journal of Medicinal Chemistry, 171,104-115. http://dx.doi.org/10.1016/j. ejmech.2019.03.031.

- Davis, C. (2019, September 18). Cancer [Web log post]. Retrieved from http://www.medicinenet.com/cancer/article.htm\#.

- $\quad$ Elsadek, B., Graeser, R., Esser, N., Schäfer-Obodozie, C., Ajaj, K. A., Unger, C. ... Kratz, F. (2010). Development of a novel prodrug of paclitaxel that is cleaved by prostate-specific antigen: An in vitro and in vivo evaluation study. European Journal of Cancer, 46(18), 3434-3444. http://dx.doi.org/10.1016/j.ejca.2010.08.018.

- Fernandez, M., Javaid, F., \& Chudasama, V. (2018). Advances in targeting the folate receptor in the treatment/imaging of cancers. Chemical Science, 9(4), 790-810. https://doi.org/10.1039/C7SC04004K.

- Florea, A. M., \& Busselberg, D., (2011). Cisplatin as an anti-tumor drug: Cellular mechanisms of activity, drug resistance and induced side effects. Cancers, 3(1), 1351-1371. doi: 0.3390/cancers3011351

- Fortin, S., \& Berube, G. (2013). Advances in the development of hybrid anticancer drugs. Expert Opinion on Drug Discovery, 8(8), 1029-1047. http://dx.doi.org/10.1517/17460441.2013.798296.

- Garraway, L. A., \& Janne, P. A. (2012). Circumventing cancer drug resistance in the era of personalized medicine. Cancer Discovery, 2, 214-226. http://dx.doi.org/10.1158/2159-8290.CD-12-0012.

- Gaspar, V. M., Costa, E. C., Queiroz, J. A., Pichon, C., \& Sousa, F. (2015). Folate-targeted multifunctional amino acid-chitosan nanoparticles for improved cancer therapy. Pharmaceutical Research, 32(2), 562-577. http://dx.doi.org/10.1007/s11095-014-1486-0.

- Gibiansky, L., \& Gibiansky, E. (2014). Target-mediated drug disposition model and its approximations for antibody-drug conjugates. Journal of Pharmacokinetics and Pharmacodynamics, 41(1), 35-47. http://dx.doi.org/10.1007/s10928-013-9344-y.

- Gupta, Y., Jain, A., Jain, P., \& Jain, S.K. (2007). Design and development of folate appended liposomes for enhanced delivery of 5-FU to tumor cells. Journal of Drug Targeting, 15(3), 231-240. http://dx.doi.org/10.1080/10611860701289719.
Guo, W., Deng, L., Chen, Z., Chen, Z., Yu, J., Liu, H. ... Zhang, L. (2018). Vitamin B12-conjugated sericin micelles for targeting CD320-overexpressed gastric cancer and reversing drug resistance. Nanomedicine, 14(3), 353-370. https://doi.org/102217/ nnm-2018-0321.

- He, R., \& Yin, C. (2017). Trimethyl chitosan based conjugate for oral and intravenous delivery of paclitaxel. Acta Biomaterial, 53, 355-366. http://dx.doi.org/10.1016/j.actbio.2017.02.012.

Henne, W. A., Kularatne, S. A., Hakenjos, J., Carron, J. D., \& Henne, K. L. (2013). Synthesis and activity of a folate targeted monodisperse PEG camptothecin conjugate. Bioorganic \& Medicinal Chemistry Letters, 23(21), 5810-5813. http://dx.doi.org/10.1016/j. bmcl.2013.08.113.

Ibsen, S., Zahavy, E., Wrasdilo, W., Berns, M., \& Chan, M. (2010). A novel doxorubicin prodrug with controllable photolysis activation for cancer chemotherapy. Pharmaceutical Research, 27(9), 1848-1860. http://dx.doi.org/10.1007/s11095-010-0183-x.

Iversen, T. G., Skotland, T., \& Sandvig, K. (2011). Endocytosis and intracellular transport of nanoparticles: Present knowledge and need for future studies. Nano today, 6(2), 176-185. http://dx.doi. org/10.1016/j.nantod.2011.02.003.

Iwata, R., Nakayama, F., Hirochi, S., Sato, K., Piao, W., Nishina, K. ... Wada, T. (2015). Synthesis and properties of vitamin E analogconjugated neomycin for delivery of RNAi drugs to liver cells. Bioorganic \& Medicinal Chemistry Letters, 25(4),815-819. http://dx.doi. org/10.1016/j.bmcl.2014.12.079.

Jung, J. H., \& Keller, T. (2012). United States (12) Patent Application Publication (10) Pub. No.: US. 310126:A1

Leamon, C. P. (2008) Folate-targeted drug strategies for the treatment of cancer. Current Opinion in Investigational Drugs, 9(12), 1277-1286. Leamon, C. P., Vlahov I. R., Reddy, J. A., Vetzel, M., Santhapuram, H. K., You, F. ... Westrick, E. (2014). Folate-vinca alkaloid conjugates for cancer therapy: A structure- activity relationship. Bioconjugate chemistry, 25(3), 560-568. http://dx.doi.org/10.1021/bc400441s. Leamon, C. P., Reddy, J. A., Vlahov, I. R., Westrick, E., Dawson, A., Dorton, R. ... Wang, Y. (2007). Preclinical antitumor activity of a novel folate-targeted dual drug conjugate. Molecular Pharmaceutics, 4(5), 659-667. https://doi.org/10.1021/mp070049c.

Mahato, R., Tai, W., \& Cheng, K. (2011). Prodrugs for improving tumortargetability and efficiency. Advanced Drug Delivery Reviews, 63(8), 659-670. http://dx.doi.org/10.1016/j.addr.2011.02.002.

- $\quad$ Naumann, R.W., \& Coleman, R. L. (2011). Management strategies for recurrent platinum-resistant ovarian cancer. Drugs, 71(11), 13971412. http://dx.doi.org/10.2165/11591720-000000000-00000.

Naumann, R. W., Coleman, R. L., Burger, R. A., Sausville, E. A., Kutarska, E., Ghamande, S. A. ... Gersh, R.H. (2013). PRECEDENT: A randomized phase II trial comparing vintafolide (EC145) and pegylated liposomal doxorubicin (PLD) in combination versus PLD alone in patients with platinum-resistant ovarian cancer. Journal of Clinical Oncology, 31(35), 4400-4406. http://dx.doi. org/10.1200/JCO.2013.49.7685.

Ojima, I., Zuniga, E., Berger, W., \& Seitz, J. (2012). Tumor-targeting drug delivery of new-generation taxoids. Future Medicinal Chemistry, 4(1), 33-50. http://dx.doi.org/10.4155/fmc.11.167.

- Padma, V. V. (2015). An overview of targeted cancer therapy. BioMedicine, 5(4). http://dx.doi.org/10.7603/s40681-015-0019-4.

Patil, S., Gawali, S., Patil, S., \& Basu, S. (2014). Synthesis, characterization and in vitro evaluation of novel vitamin D3 nanoparticles as a versatile platform for drug delivery in cancer therapy. Journal of Material Chemistry B, 5742. https://doi.org/10.1039/C3TB21176B. Palvai, S., Nagraj, J., Mapara, N., Chowdhury, R., \& Basu, S. (2014). Dual drug loaded vitamin D3 nanoparticle to target drug resistance in cancer. RSC Advances, 4(100), 57271-57281. http://dx.doi. org/10.1039/C4RA06475E. 
- Parhi, P., Mohanty, C., \& Sahoo, S. K. (2012). Nanotechnologybased combinational drug delivery: an emerging approach for cancer therapy. Drug Discovery Today, 17(17-18), 1044-1052. http://dx.doi.org/10.1016/j.drudis.2012.05.010.

- Pawar, S. K., Badhwar, A. J., Kharas, F., Khandare, J. J., \&Vavia, P. R. (2012). Design, synthesis and evaluation of $\mathrm{N}$-acetyl glucosamine (NAG)-PEG-doxorubicin targeted conjugates for anticancer delivery. International Journal of Pharmaceutics, 436(1-2),183-193. http://dx.doi.org/10.1016/j.ijpharm.2012.05.078.

- Pérez-Herrero, E., \& Fernández-Medarde, A. (2015). Advanced targeted therapies in cancer: drug nanocarriers, the future of chemotherapy. European Journal of Pharmaceutics and Biopharmaceutics, 93, 52-79. http://dx.doi.org/10.1016/j.ejpb.2015.03.018.

- Persidis, A. (1999). Cancer multidrug resistance. Nature Biotechnology, 17(1), 94. http://dx.doi.org/10.1038/5289.

- $\quad$ Petros, R. A., \& DeSimone, J. M. (2010). Strategies in the design of nanoparticles for therapeutic applications. Nature Reviews Drug discovery, 9(8), 615. http://dx.doi.org/10.1038/nrd2591.

- Pettenuzzo, A., Pigot, R., \& Ronconi, L. (2017). Vitamin B12-metal conjugates for targeted chemotherapy and diagnosis: Current status and future prospects. European Journal of Inorganic Chemistry, (12), 1625-1638. https://doi.org/10.1002/ejic.201601217.

- Pinhassi, R. I., Assaraf, Y. G., Farber, S., Stark, M., Ickowicz, D., Drori, S. ... Livney, Y. D. (2009). Arabinogalactan-folic acid-drug conjugate for targeted delivery and target-activated release of anticancer drugs to folate receptor-overexpressing cells. Biomacromolecules, 11(1), 294-303. http://dx.doi.org/10.1021/bm900853z.

- Qu, C.Y., Zhou, M., Chen, Y. W., Chen, M. M., \& Shen, F. (2015). Engineering of lipid prodrug-based, hyaluronic acid-decorated nanostructured lipid carriers platform for 5-fluorouracil and cisplatin combination gastric cancer therapy. International Journal of Nanomedicine, 10, 3911. http://dx.doi.org/10.2147/IJN.S83211.

- Rajendran, L., Knolker, H. J., \& Simons, K. (2010). Subcellular targeting strategies for drug design and delivery. Nature Reviews Drug Discovery, 9(1), 29. http://dx.doi.org/10.1038/nrd2897.

- Reddy, J. A., Dorton, R., Westrick, E., Dawson, A., Smith, T., Xu, L. C. ... Leamon, C.P. (2007). Preclinical evaluation of EC145, a folatevinca alkaloid conjugates. Cancer Research, 67(9), 4434-4442. http://dx.doi.org/10.1158/0008-5472.CAN-07-0033.

- Reddy, J. A., Dorton, R., Dawson, A., Vetzel, M., Parker, N., Nicoson, J.S. ... Leamon, C.P. (2009). In vivo structural activity and optimization studies of folate- tubulysin conjugates. Molecular Pharmaceutics, 6(5), 1518-1525. https://doi.org/10.1021/mp900086w.

- Rijnboutt, S., Jansen, G., Posthuma, G., Hynes, J. B., \& Schornagel, J. H. (1996). Endocytosis of GPI-linked membrane folate receptoralpha. The Journal of Cell Biology, 132(1), 35-47. http://dx.doi. org/10.1083/jcb.132.1.35.

- Ruiz-Sánchez, P., König, C., Ferrari, S., \& Alberto, R. (2011). Vitamin B 12 as a carrier for targeted platinum delivery: In vitro cytotoxicity and mechanistic studies. Journal of Biological Inorganic Chemistry, 16(1), 33-44. http://dx.doi.org/10.1007/s00775-010-0697-z.
Russell-Jones, G., McTavish, K., \& McEwan, J. (2011). Preliminary studies on the selective accumulation of vitamin-targeted polymers within tumors. Journal of Drug Targeting, 19(2), 133-139. http://dx.doi.org/10.3109/10611861003734027.

Sausville, E., LoRusso, P., Quinn, M., Forman, K., Leamon, C., Morganstern, D. ... Messmann, R. (2007). A phase I study of EC145 administered weeks 1 and 3 of a 4-week cycle in patients with refractory solid tumors. Journal of Clinical Oncology, 25(18_suppl), 2577. http://dx.doi.org/10.1200/JCO.2011.41.4946.

- Seifu, M. F., \& Nath, L. K. (2019). Polymer-drug conjugates: novel carriers for cancer chemotherapy. Polymer-Plastics Technology and Materials, 58(2), 1158-1171. https://doi.org/10.1080/036255 9.2018.1466172.

- Seitz, J. D., Vineberg, J. G., Herlihy, E., Park, B., \& Melief, E. (2015). Design, synthesis and biological evaluation of a highly potent and cancer cell selective folate-taxoid conjugate. Bioorganic \& Medicinal Chemistry, 2187-2194. http://dx.doi.org/10.1016/j.bmc.2015.02.057.

- $\quad$ Siega, P., Wuerges, J., Arena, F., Gianolio, E., Fedosov, S.N., Dreos, R. ... Randaccio, L. (2009). Release of toxic Gd3 + ions to tumour cells by vitamin B12 bioconjugates. Chemistry-A European Journal, 15(32),7980-7989. http://dx.doi.org/10.1002/chem.200802680.

- $\quad$ Sutherland, J. C., \& Griffin, K. P. (1981). Absorption spectrum of DNA for wavelengths greater than $300 \mathrm{~nm}$. Radiation Research, 86(3), 399-410. http://dx.doi.org/10.2307/3575456.

- Tran, M. T., Stürup, S., Lambert, I. H., Gammelgaard, B., \& Furger, E. (2016). Cellular uptake of metallatedcobalamins. Metallomics, 8(3), 298-304. http://dx.doi.org/10.1039/c5mt00272a.

- $\quad$ Tripodo, G., Mandracchia, D., Collina, S., Rui, M., \& Rossi, D., (2014). New perspectives in cancer therapy: the biotin-antitumor molecule conjugates. Medicinal Chemistry, 8, 1-4. http://dx.doi. org/10.4172/2161-0444.S1-004.

- Vlahov, I. R., \& Leamon, C. P. (2012). Engineering folate-drug conjugates to target cancer: from chemistry to clinic. Bioconjugate Chemistry, 23(7),1357-1369. http://dx.doi.org/10.1021/bc2005522.

- Vlahov, I. R., Leamon, C. P., Parker, M. A., Howard, S. J., Santhapuram, H. K., Satyam, A., \& Reddy, J. A. (2009). Inventors; Endocytelnc, assignee. Vitamin receptor binding drug delivery conjugates, United States patent US 7,601,332.

- Wang, X., Li, J., Wang, Y., Koenig, L., Gjyrezi, A., Giannakakou, P. ... Shin, D. M. (2011). A folate receptor-targeting nanoparticle minimizes drug resistance in a human cancer model. ACS Nano, 5(8), 6184-6194. http://dx.doi.org/10.1021/nn200739q.

- Wang, Y., Li, P., Chen, L., Gao, W., \& Zeng, F. (2015). Targeted delivery of 5-fluorouracil to HT-29 cells using high efficient folic acidconjugated nanoparticles. Drug Delivery, 22(2), 191-198. http:// dx.doi.org/10.3109/10717544.2013.875603.

- Yuxuan, D., Xingguang, C., Xinzhou, B., Chunxia L., Na, Y., Ying, Z., Jiaqi, Z., \& Mian, F. (2019). Synthesis and anti-cancer evaluation of folic acid-peptide- paclitaxel conjugates for addressing drug resistance. European Journal of Medicinal Chemistry, 171, 104-115. doi: 10.1016/j.ejmech.2019.03.031 